\title{
The Treatment of a Patient Who Is Also a Mental Health Practitioner: Special Considerations and Recommendations
}

\author{
Miriam Goldberg $^{1} \cdot$ Renana Stanger Elran ${ }^{2} \cdot$ Yael Mayer $^{3} \cdot$ Ido Lurie $^{4}$ (D)
}

Received: 29 April 2020 / Accepted: 25 June 2020 / Published online: 24 July 2020

(C) Academic Psychiatry 2020

The first time we met (MG \& IL, authors) was as guest speakers at a seminar for social work students at an Israeli university, intended to expose them to the field of mental health. The next time we met was in an inpatient psychiatric department, as a patient (MG) and the head of the department (IL). About a year later, we decided, with colleagues (RSE, YM), to put our previous interactions, perspectives, personal, and professional knowledge into a joint paper regarding the treatment of colleagues within the mental health system.

Treating a colleague, a mental health practitioner with a mental disorder, is almost unspoken of, despite the prevalence of mental disorders and high levels of distress among practitioners $[1,2]$. Furthermore, the choice of becoming a mental health professional may be influenced by psychological crises, personal loss, and mental health difficulties, whether personally or in the near environment [3,4]. The "wounded healer" archetype refers to this duality when therapists themselves face mental difficulties and turn their own pain into strength and ability to help others [5]. Both the therapist and therapatient (a therapist who is also a patient; [6]) face unique and complex challenges in this inter-personal encounter, including questions relating the personal and professional identity, ethical dilemmas, and professional issues.

The purposes of this paper are to illuminate the topic, review and describe the barriers for seeking treatment among mental health practitioners, the complexities and challenges of treating a therapatient and suggestions and recommendations.

Ido Lurie

ido.lurie@gmail.com

1 Bar-Ilan University, Ramat Gan, Israel

2 Hebrew University, Jerusalem, Israel

3 The University of British Columbia, Vancouver, Canada

4 Tel Aviv University, Tel Aviv, Israel

\section{Emotional Distress and Psychiatric Morbidity Among Mental Health Practitioners}

No one is immune to mental disorder. In fact, medical practitioners - at all stages of training - are at increased risk of burnout, mental distress, and psychiatric disorders, with personal and occupational risk factors $[7,8]$. In a study of teams in four National Health Service Centers (NHS, $N=$ 2073), of which $65 \%$ were staff members in the health and welfare professions, $48 \%$ stated that they had dealt with a mental health condition [7]. Also, 20\% stated that they were facing a mental health condition at the time of the study. The most common mental disorders reported were depression $(n=$ $632,49 \%)$, anxiety ( $n=461,35.7 \%)$, as well as posttraumatic stress disorder $(n=47,3.6 \%)$, obsessive-compulsive disorder $(n=39,3.02 \%)$, and psychosis and personality disorders $(n=$ $25,1.94 \%$, each of the disorders) [7].

In a systematic review and meta-analysis of burnout and mental distress among mental health practitioners (33 studies, $N=9409$ ), signs of burnout (emotional exhaustion, depersonalization, low sense of ability) were diagnosed among 20 $40 \%$ of the participants [2]. A personal risk factor for burnout was older age, though it was also associated with a higher sense of personal satisfaction, which is a protective factor. Risk factors related to the work environment include workload and work relationships. Protective factors were role clarity, sense of professional autonomy, being treated fairly, receiving fair reward for one's work, and access to regular clinical training. Workers of mental health teams in the community were at greater risk of burnout compared to communitybased specialist teams [2].

The suicidality rate among US physicians was found to be 28-40 per 100,000, twice the rate in the general population [9]. A meta-analysis of suicidality risk among physicians found that physicians are at an increased risk of suicidality, women at a more increased risk (1.9) than men, with psychiatry being one of the specialties at increased risk. About $17 \%$ of the physicians had suicidal thoughts [10]. 


\section{Barriers for Seeking Treatment Among Mental Health Practitioners}

Mental health practitioners face unique barriers for seeking treatment. In a study of 260 APA member psychologists, approximately $86 \%$ reported that they had been in psychotherapy at some point in their lives, and it has been 12.7 years on average (SD 11.2) since their last session. Alarmingly, $60 \%$ reported that there was a point in their lives when they felt they could benefit from psychotherapy yet did not seek it [11].

Based on studies, there are a several factors that may lead mental health practitioners to avoid mental health treatment. Firstly, there is the concern that colleagues will find out about the mental disorder, and unwillingness to disclose such issues. This involves a realistic concern regarding the implications for professional practice (e.g., the possible legal requirement to report potential risk for patients), to the point of risking one's potential career. Based on social stigma and self-stigma [12], treatment-seeking may be portrayed and perceived as a sign of weakness and dysfunction. This may be related to the covert assumption that working in a therapeutic profession confers immunity to coping with a mental disorder [13, 14]. Therapists have difficulty finding another therapist, especially when they are members of small professional communities of mental health care. In addition, therapists may have difficulties in finding time for treatment due to workload and low focus on personal self-care $[11,15]$.

\section{Complexities and Challenges: the Perspective of the Therapatient's Therapist}

Based on our own professional experiences and observations, treating a therapatient can summon challenging situations for therapists. Within the therapeutic setting, due to the understanding that the client is a colleague familiar with professional jargon, and possible unpleasantness, shame, and reluctance to make comprehensive questioning (e.g., regarding sexual trauma, use of psychoactive substances), the interview may not be conducted as fully and openly as needed. This may lead to partial or incorrect formulation, diagnosis, and treatment. In addition, out of empathy and concern, the treatment can lead to the therapist's over-identification with the therapatient, over-evaluating strengths and resources, ignoring premature symptoms, and preventing accurate and timely treatment.

Furthermore, the therapatient may go through periods of worsening mental state, suicidality, impaired functioning, and judgement, all of which might challenge the professional loyalty, discretion, and medical confidentiality towards the therapatient and his/her patients. The therapist needs to assess the therapatient's ability to continue practicing his/her profession and has a duty to report when there is risk to the therapatients' patients, to the point of violating the autonomy of the therapatient. The therapatient may be very aware of the therapist's different roles and responsibilities, and therefore conceal important information, regarding dysfunctional parts of the self from the therapist.

The treatment of a therapatient can cause blurred boundaries, such as ambiguities between therapy, supervision, and employment. Outside of the therapeutic setting, situations of professional meetings may occur, sometimes unplanned (e.g., conferences, lectures), which may cause embarrassment.

\section{Complexities and Challenges: the Therapatient's Point of View}

Illness is the night side of life, a more onerous citizenship. Everyone who is born holds dual citizenship, in the kingdom of the well and in the kingdom of the sick. Although we all prefer to use the good passport, sooner or later, each of us is obliged, at least for a spell, to identify ourselves as citizens of that other place. [16, p.3]

Being a therapatient poses an identity challenge. Deegan [17] referred to an accepted perception of "us" vs "them", those diagnosed with a mental disorder. This dichotomous separation allows the creation of an artificial reality of a normal healthy world as opposed to a non-normal, ill world. This approach persists in the mental health field between "us" health professionals, who are perceived as healthy, competent, and high functioning, vs "them"- the patients, who are depicted as sick, needy, and weak. A tangible example of the symbolic "glass wall" is the nursing station in many psychiatric wards, which is actually surrounded by a glass wall, referred to by the patients as "the aquarium". The therapatient challenges this dichotomy and is forced to fight for legitimacy to practice in mental health professions and to hold "citizenship" in them $[18,19]$.

In addition, the therapatient needs to deal with the transitions between different spaces: personal, therapeutic, and collegial. For example, issues that come up in therapy may relate to personal challenges, but also to professional ones, such as experiences with patients, thus blurring the distinction between personal therapy and work-related professional issues. The therapatient should feel legitimized and free to bring in emotional needs or dysfunctional parts of the self within the treatment without feeling that the therapist is judgmental.

Another challenge is the formulation of a professional identity, including how the therapatient integrates his/her identity roles - both as a service provider and as a service user. The therapatient may consider the ways the mental disorderrelated identity enhances or complicates the professional 
identity. Another possible setting issue may arise in cases when the treatment is in a public clinic or psychiatric ward, causing a sense of "forced exposure" or "outing".

\section{Recommendations for Addressing Possible Complexities in Treating a Therapatient}

This section does not constitute definitive guidelines but rather suggestions that we formulated based on our observations and our discussions, between therapists and therapatients with the desire to expand the discourse. In general, it is recommended that there will be room to discuss arising challenges related to the unique encounter (e.g., fear that competence will be questioned; consequences of stigma and self-stigma; forced disclosure). Below are a few suggestions to consider when treating a therapatient:

\section{Help in Role-Identity Formation and Self-disclosure}

The need to clarify feelings and concerns in the face of dual identity as a therapatient is extremely important. Since selfdisclosure is a continuous and dynamic process, the therapist could encourage therapatients to explore the different strategies for disclosing their mental health disorder, in ways that are beneficial for their well-being and identity formation [20]. This process involves considering ramifications that may stem from self-disclosure, including risk management, requesting accommodations in the workplace, discussing the fear of stigma, professional delegitimization, as well as experiences with supervisors, colleagues, and patients.

The therapist can help the therapatient choose the degree of desired disclosure, in relation to personality, stage in life, coping with the disorder, and professional development. It could be meaningful to examine how life experiences acquired through coping with mental disorder add and enrich the professional identity, or make it difficult to treat others (e.g., overidentification with patients, facing emotional triggers in the workplace) $[6,21]$. The goal is to help the therapatient in the process of finding his/her unique personal and professional voice or stance, while feeling confident in the ability to control disclosure processes.

\section{Setting and Ensuring Discretion Beyond Medical Confidentiality}

Sometimes, careful attention and extra discretion, beyond the obvious medical confidentiality, are in need. It is important to have a preliminary discussion as part of building the therapeutic setting and to coordinate in advance how to manage "sensitive" situations (e.g., meeting in collegial circumstances). Some therapatients will prefer not to identify as patients, so although it is not possible to avoid opening a patient file in a public clinic, the appointments can be held at certain hours in which it is less likely to meet other staff members or patients. The therapist and therapatient can decide on creating cover-up stories explaining the purpose of the meeting to other staff members (e.g., training, joint project, research) to help therapatients keep confidentiality of treatment, when needed.

\section{Boundaries Versus Flexibility}

There should be a delicate interplay between boundaries and flexibility in the treatment of therapatients. Occasionally, extra flexibility is needed, for example, in setting meeting hours, choosing the place of treatment. Yet, it is also important to maintain boundaries and maintain the setting, for example, avoiding "corridor medicine" in order to sustain the treatment's boundaries and safety.

\section{Examining the Preliminary Circumstances Before Treatment}

Before starting treatment, both sides should consider if there are overlapping social-collegial circles that may impair treatment. The therapatient could be asked to make an informed choice of therapist, and if there is too much overlap in the circles, treatment by a certain therapist can be avoided.

\section{Transparency and Sharing}

It is important to allow space for the therapatient's professional sides and to share dilemmas that arise with transparency and respect to the functional and knowledgeable parts of the therapatient, without under-rating the difficulty of the mental challenge.

\section{Encourage Self-care}

Attention should also be paid to returning responsibility to the hands of the therapatient, so that s/he may rely on existing inner resources. It is also advisable to help the therapatient redirect existing personal resources to self-care. A self-care program can be built and integrated into the busy professional life in order to prevent burnout.

\section{Personal and Professional Responsibility}

The therapatient should be encouraged to think about personal commitment and responsibility for treating him/herself, which is critical to the ability to treat others, and in fact is part of a professional responsibility [6]. Thus, overseeing the professional ability of the therapatient will not only be in the hands of external parties, such as medical committees, but will become a personal responsibility of the therapatient him/herself. 


\section{Identifying Incapacity and Providing Adequate Support}

The therapist should provide the therapatient with adequate help in situations of incapacity that compromise the professional ability to treat patients, as well as guidance and support in returning to work after the crisis is over. It is important that the process will be done with care, sensitivity, and by collaborative dialog, while emphasizing the personal agency of the therapatient, and encouraging him/her to make decisions.

\section{The Role of Academia}

Academia has a role to play in promoting awareness and knowledge on both the merits and complexities of being a mental health practitioner coping with mental disorder, as well as on the dilemmas that arise when treating such patients. The healthcare curriculum should include references to the growing body of knowledge and research on lived experience of people coping with mental disorders. Specifically, students and interns should be exposed to first-person accounts of mental health practitioners coping with mental disorders and learn from their experiential knowledge [22]. The exposure to these topics can contribute to the self-awareness and self-reflection of the students regarding their own difficulties [23], and also enhance their feelings of empathy towards both patients and colleagues. Further benefits may also be combating social and self-stigma among mental health practitioners, and legitimizing seeking treatment and emotional support throughout different stages of training.

\section{Discussion}

The therapatient copes with mental disorder, but at the same time also develops as a mental health professional [6], acquiring both professional and experiential knowledge. The Jungian archetype of the "Wounded healer" illuminates the recognition that therapatients can have important and unique contributions to the mental health field. Due to their inside knowledge and experience, they can understand their patients' perspectives in a nuanced and embodied way. Thus, mental health practitioners coping with mental disorders bring rich and valuable personal experience, for both their patients and for the profession itself. Therefore, therapatients can have an important impact on improving the quality of care in mental health systems. In addition, therapatients' work may help in breaking the "glass wall" between the "us vs them" dichotomy and balance the power dynamics that exist within health systems - between patients and therapists - while promoting a more open dialog that can only exist when there is more equality between the parties. Finally, therapatients can serve as important role models of coping, recovery, and hope for their patients and fellow colleagues.

\section{Compliance with Ethical Standards}

Disclosures On behalf of all authors, the corresponding author states that there is no conflict of interest..

\section{References}

1. Dattilio FM. The self-care of psychologists and mental health professionals: a review and practitioner guide. Aust Psychol. 2015;50: 393-9.

2. O'Connor K, Muller Neff D, Pitman S. Burnout in mental health professionals: a systematic review and meta-analysis of prevalence and determinants. Eur Psychiatry. 2018;53:74-99.

3. Barnett M. What brings you here? An exploration of the unconscious motivations of those who choose to train and work as psychotherapists and counsellors. Psychodyn Pract. 2007;13:257-74.

4. Farber BA, Manevich I, Metzger J, Saypol E. Choosing psychotherapy as a career: why did we cross that road? J Clin Psychol. 2005;61:1009-31.

5. Zerubavel N, Wright MO. The dilemma of the wounded healer. Psychother Theory Res Pract Train. 2012;49:482-91.

6. Goldberg M, Hadas-Lidor N, Karnieli-Miller O. From patient to therapatient: social work students coping with mental illness. Qual Health Res. 2015;25:887-98.

7. Sherring S. Mental illness among NHS health care workers: a survey. Br J Ment Heal Nurs. 2019;8:129-35.

8. Kottsieper P. Wounded healers need not apply. In: Swarbrick P, Schmidt LT, editors. People recover as Provid Psychiatr Rehabil build wisdom Exp. Linthicum: United States Psychiatric Rehabilitation Association; 2010.

9. Anderson P. Physicians experience highest suicide rate of any profession [Internet]. Medscape. 2018 [cited 2020 Apr 21]. Available from: https://www.medscape.com/viewarticle/896257

10. Dutheil F, Aubert C, Pereira B, Dambrun M, Moustafa F, Mermillod M, et al. Suicide among physicians and health-care workers: a systematic review and meta-analysis. PLoS One. 2020;14.

11. Bearse JL, McMinn MR, Seegobin W, Free K. Barriers to psychologists seeking mental health care. Prof Psychol Res Pract. 2013;44: $150-7$.

12. Nemec PB, Swarbrick M, Legere L. Prejudice and discrimination from mental health service providers. Psychiatr Rehabil J. 2015;38: 203-6.

13. Tillett R. The patient within - psychopathology in the helping professions. Adv Psychiatr Treat. 2003;9:272-9.

14. Sherman MD. Distress and professional impairment due to mental health problems among psychotherapists. Clin Psychol Rev. 1996;16:299-315.

15. Edwards JL, Crisp DA. Seeking help for psychological distress: barriers for mental health professionals. Aust J Psychol. 2017;69: $218-25$.

16. Sontag S. Illness as metaphor and AIDS and its metaphors. New York: Anchor Books; 1990.

17. Deegan PE. Recovery: the lived experience of rehabilitation. Psychosoc Rehabil J. 1988;11:11-9.

18. Easterbrook A, Bulk L, Ghanouni P, Lee M, Opini B, Roberts E, et al. The legitimization process of students with disabilities in health and human service educational programs in Canada. Disabil Soc. 2015;30:1505-20. 
19. Bulk LY, Easterbrook A, Roberts E, Groening M, Murphy S, Lee $\mathrm{M}$, et al. 'We are not anything alike': marginalization of health professionals with disabilities. Disabil Soc. 2017;32:615-34.

20. Bulk LY, Tikhonova J, Gagnon JM, Battalova A, Mayer Y, Krupa $\mathrm{T}$, et al. Disabled healthcare professionals' diverse, embodied, and socially embedded experiences. Adv Heal Sci Educ. 2020;25:11129.

21. Mayer, Y., Jarus, T., Gross, E., Bulk, L., Nimmon, L., Krupa, T., Murphy, S., Shalev, M., Battalova, A., Lee, M., Copland, A., Khairati, F., \& Parhar G. Disabled and competent: professional identity formation processes of healthcare students and clinicians with disabilities. CHES (Centre Heal Educ Sch Celebr Scholarsh Conf [Internet]. Vancouver, British Columbia; 2019. Available from: https://med-fom-osot-inclusive-campus.sites.olt.ubc.ca/files/ 2019/09/Identity-poster.pdf. (Last accssed 30 May, 2020).

22. Kraus E, Moran G. When social work students meet workers with mental-health lived-experience: a case study. J Soc Work Educ. 2019;38:861-74.

23. Weerman A, Abma T. Social work students learning to use their experiential knowledge of recovery. An existential and emancipatory perspective. J Soc Work Educ. 2018.

Publisher's Note Springer Nature remains neutral with regard to jurisdictional claims in published maps and institutional affiliations. 